\title{
Annular Bright-Field Electron Microscopy Tracking Solid-State Chemical Reaction
}

\author{
Peng Gao ${ }^{1}$, Ryo Ishikawa ${ }^{1}$, Eita Tochigi ${ }^{1}$, Akihito Kumamoto ${ }^{1}$, Naoya Shibata ${ }^{1}$, Yuichi Ikuhara ${ }^{1}$ \\ ${ }^{1}$ Institute of Engineering Innovation, School of Engineering, The University of Tokyo, Tokyo 113-8656, \\ Japan
}

To track the structural evolution during solid-state chemical reactions and phase transformations, in situ $\mathrm{X}$-ray scattering, nuclear magnetic resonance and electron microscopy are the most commonly used techniques. Although the bulk-based measurement methods enable valuable insights into the evolution of long-range structure, they have inadequate spatial resolution to probe phase boundaries propagation or localized atomic displacements. The atomic number (Z)-contrast high angle annular dark-field (HAADF) in aberration corrected scanning transmission electron microscopy (STEM) makes it possible to capture phase boundaries motion at atomic resolution [1] and track individual atoms migration in realtime [2], but it is almost blind to the light atoms (e.g., lithium) in chemical compounds. In contrast, the recent advancements in the annular bright field (ABF) imaging [3], allow us to quantitatively determine the composition and occupancy of both heavy and light elements columns, providing a unique and powerful tool/technique to study the structures of functional materials and dynamics processes therein.

Here we demonstrate an approach based on ABF electron microscopy to visualize a reduction reaction driven by electron beam radiolysis in manganese oxides. Spinel $\mathrm{Mn}_{3} \mathrm{O}_{4}$ has three different $\mathrm{Mn}$ columns (Fig.1a) and two O columns (Fig.1b). Mn1 columns are much brighter in Z-contrast image Fig. 1a because they contain twice as many cations as Mn2 and Mn4 columns. The O1 columns should be slightly misaligned based on the spinel structure and lean to Mn1 sites as shown in Fig. 1b. As a transition-metal oxide, it's well known that the incident electron beam in electron microscope can knock oxygen out of manganese oxide (via Knotek-Feibelman mechanism), leading to reduction. After exposure to electron beam for a while, spinel structure frame with high concentration of oxygen vacancies becomes unstable. Meanwhile, gained energy from the incident electron beam, the cations become very active and hence the structure tends to convert into rock salt with less nonstoichiometry. Partial cations at Mn4 sites gradually fill the vacant Mn3 columns indicated by the arrows in the Fig. 1c. The other Mn4 atoms fill the Mn2 columns as in a rock salt structure all the cation columns have the same intensity. During cations migration, the oxygen anions shift accordingly. In the rock salt structure, the anions prefer to stay at symmetric positions that are the middle points of two neighboring cations. Therefore, upon reduction O1 columns shift away from Mn1 and the distance of two O1 columns (indicated in the Fig.1b) shortens. Fig.1d shows the distance is decreased by $\sim 1.2 \%$ every 10 seconds, equating to an averaged velocity $\sim 0.35 \mathrm{pm} / \mathrm{s}$ for $\mathrm{O} 1$ columns shift. The reduction firstly occurs at the surface where the oxygen ions can easily be released and then gradually propagates into interior with further exposure. The reaction front planes (i.e., phase boundaries between reduced surfaces and pristine core) that are perpendicular to the viewing direction are also identified from the ABF image.

This methodology demonstrated herein enables real-time tracking full atoms motions during a reduction of manganese oxides. Thanks to the high sensitivity to both light and heavy atom columns, the ABF imaging can achieve unprecedented details of dynamic processes. With further improved stability of environmental sample holders in future, ABF electron microscopy will ultimately allow various in situ studies of solid state transformation and electrochemical/chemical reactions at atomic resolution and lead to beneficial technological solutions to industry [4]. 
References:

[1] P. Gao et al, Nature Communications 4 (2013) 2791.

[2] R. Ishikawa et al, Physical Review Letters, 113 (2014) 155501.

[3] S. Findlay et al, Applied Physics Express, 3 (2010) 116603.

[4] The authors gratefully acknowledge the financial support through a Grant-in-Aid for Scientific Research on Innovative Areas "Nano Informatics" (Grant No. 25106003) from Japan Society for the Promotion of Science (JSPS), and "Nanotechnology Platform" (Project No. 12024046) from the Ministry of Education, Culture, Sports, Science and Technology in Japan (MEXT).

a

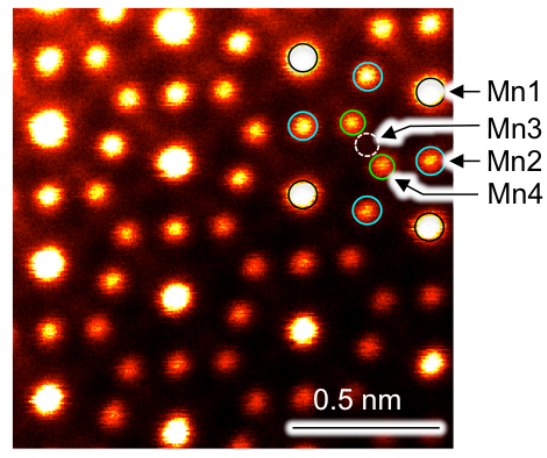

b

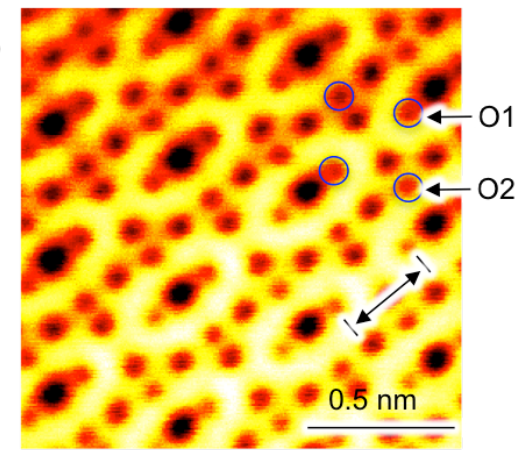

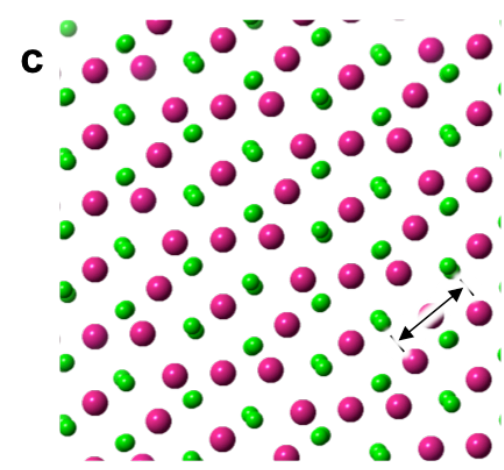
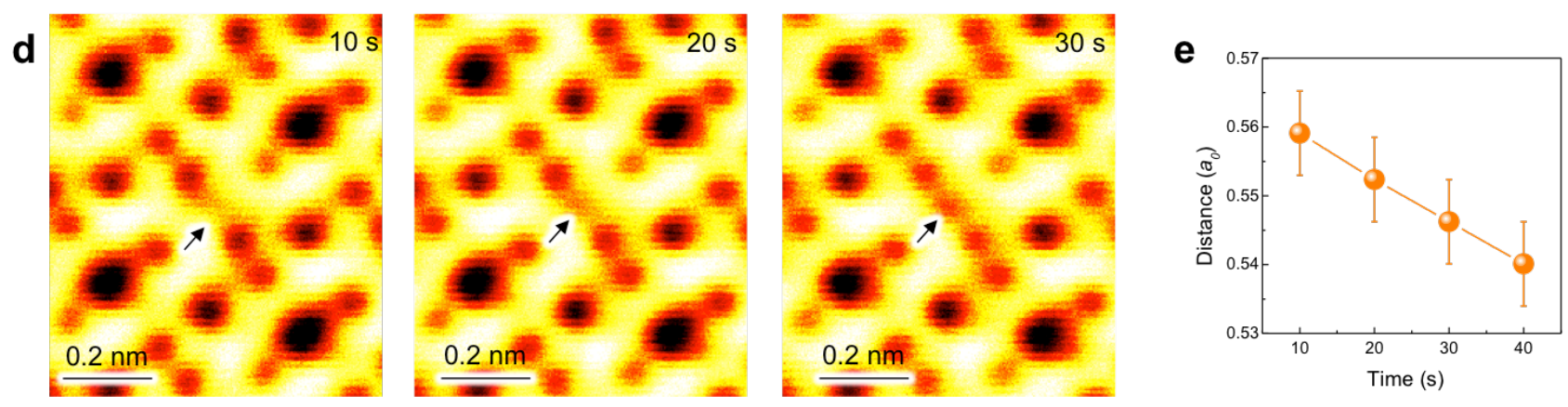

Figure 1. High resolution HAADF (a) and simultaneously recorded ABF (b) images of $\mathrm{Mn}_{3} \mathrm{O}_{4}$ particle from a cold FEG GRAND ARM at $300 \mathrm{kV}$ with spatial resolution of $45 \mathrm{pm}$. Four types of cations columns and two types of anions columns are labeled respectively. The distance between two O1 columns is calculated to measure the shift of $\mathrm{O}$ during reduction. (c) The atomistic model illustrating spinel $\mathrm{Mn}_{3} \mathrm{O}_{4}$ structure along the viewing direction of [111]. Purple: Mn. Green: O. (d) Sequential series of $\mathrm{ABF}$ images showing the spinel $\mathrm{Mn}_{3} \mathrm{O}_{4}$ converts into rock salt $\mathrm{MnO}$ and $\mathrm{O}_{2}$. The arrows indicating the Mn3 sites are gradually filled. (e) The distance between two O1 columns [indicated in (b)] is reduced $\sim 3.5 \%$ within $40 \mathrm{~s}$, corresponding to $\sim 28 \mathrm{pm}$. 